\title{
Pet therapy con adolescenti nel contesto familiare
}

\begin{abstract}
Aluette Merenda ${ }^{l}$
RIASSUNTO

L'autore si propone di delineare il valore terapeutico degli animali domestici nell'ambiente familiare e nei contesti di cura, rintracciandone risvolti psicoeducativi e finalità terapeutiche. In particolare, entro una prospettiva evolutivo-familiare e nell'ambito dell'approccio zooantropologico, centrato sul processo interattivo uomo-animale, si configurano le caratteristiche di un contesto di cura che tiene conto del benessere psicofisico e delle peculiarità di coloro che vengono coinvolti in un incontro etero-specifico ed in cui viene valorizzata una co-partnership, nella sua valenza terapeutica. Partendo dal presupposto che gli animali d'affezione non siano fautori di miracolose guarigioni, si vuole porre l'accento sulle attività relazionali assistite dalla presenza di due co-terapeuti (uomoanimale), finalizzate a benefici di ordine educativo e riabilitativo, nonché mirate all'attraversamento del rischio individuale e familiare o al trattamento di psicopatologie.

Il focus dell'articolo si orienta specificatamente su una serie di dimensioni: Pet therapy e Zooantropologia nelle famiglie attuali; Pet

\footnotetext{
${ }^{1}$ Psicologa, psicoterapeuta della Gestalt, ricercatore universitario in Psicologia dinamica presso la Facoltà di Scienze della Formazione, Università degli Studi di Palermo. Afferenza: Dipartimento di Psicologia di Palermo, tlf+39 09123897731; Email: aluette.merenda@unipa.it.

Questo contributo fa riferimento alla long version di un paper presentato alla First Italian Conference on Society and animals: new perspective on human and nature, Palermo, IZS, 13-14 Marzo, 2009.
} 
relationships e questioni metodologiche; Pet relationship come incontro etero-specifico in adolescenza.

Parole chiave: Pet relationships, Zooantropologia, Pet therapy, Pet pedagogy, nuove famiglie, contesto di cura;

\section{SUMMARY}

Pet-relationships and family care settings: a hetero-specific meeting in adolescence.

In this article, the Author aims to delineate the therapeutic value of domestic animals (Pets) in the family environment and in care settings, tracking psycho-educational aspects as well as therapeutic objectives. In particular, within a familiar developmental model and in the zooanthropology approach, which considers the interactive process between animals and humans, the study outlines the characteristics of a care setting as well as those therapists who are involved in a hetero-specific meeting, in which a co-partnership and its therapeutic value is emphasised.

On the assumption that pets do not constitute medication nor are they supporters of miraculous cures, the Author focuses on Pet Relationship Activities assisted by two co-therapists (human and animal), aimed at the education on policy and rehabilitation benefits, as well as at the cross over between individual and family risk or at psychopathological treatment.

The focus of the article is specifically directed at several dimensions: Pet therapy and Zooanthropology in new families; Pet relationship and its methodological issues and perspectives; Pet relationship as a hetero-specific meeting in adolescence.

Keywords: Pet relationship, Zooanthropology, Pet therapy, Pet pedagogy, new families, care settings. 


\section{Pet Therapy e ZooAntropologia nelle famiglie attuali}

«Una casa e una famiglia non sono casa e famiglia senza un pet» (Bettetini, 2006, p.25)

Nell'ambito delle tipologie familiari presenti nell'attuale panorama sociale postmoderno si registra una popolazione animale che suscita interesse: circa 310 milioni di animali domestici risulterebbero presenti nelle case europee, di cui oltre 60 milioni in Italia (in particolare: 14 milioni tra cani e gatti $)^{2}$. Una presenza così numerosa può indurre a molteplici riflessioni, estendibili in un'ottica di epistemologia familiare verso la configurazione di un ulteriore scenario di famiglia: «allargata, mista con animali familiari») (Ballarini, 2000,p.19).

Tale tipologia, che va oltre la famiglia esclusivamente "umana", riguarderebbe infatti nuclei familiari misti, in cui un animale domestico o familiare assume funzioni plurime e peraltro circoscrivibili nell'ambito delle nuove forme o tendenze attuali del vivere insieme ${ }^{3}$ : unipersonali (singles, anziani), monoparentali, adottive (e/o affidatarie), coppie childless e childfree.

Più dettagliatamente, nelle famiglie unipersonali, quali forme familiari configurate dalla massima contrazione familiare, l'evento normativo del viver soli ma in co-presenza di un animale domestico assume significati evolutivi specifici: per una scelta di vita transitoria, che può caratterizzare ad esempio quella di un giovane adulto che si confronta da un lato con la propria capacità di separazione-individuazione dal nucleo familiare d'origine e altresì con la propria competenza nel prendersi cura di un Altro diverso da sé; o inoltre, per l'ultima transizione del ciclo vitale, in cui gli animali d'affezione assumono il compito evolutivo di alleviare quei

\footnotetext{
2 Per ulteriori approfondimenti sulle tendenze principali ed attuali legate al ruolo degli animali da compagnia nelle famiglie e nella società si vedano: il Rapporto Assalco 2009 e Rapporto Assalco - Zoomark 2011; Rapporto Eurispes 2010 del Ministero della Salute. ${ }_{3}^{3}$ Per un approfondimento sulle trasformazioni delle tipologie familiari si veda: Salerno A.(2010). Vivere insieme. Tendenze e trasformazioni della coppia moderna. Bologna: Il Mulino.
} 
vissuti di angoscia, solitudine, abbandono e decadimento psicofisico, spesso intensamente sperimentati dalla famiglia in età anziana. In altri termini, come mediatrice di abilità psicosociali, la presenza nella vita domestica ed affettiva di un pet ${ }^{4}$ può offrire una serie di spunti di benessere psicoemotivo : «stimolando la motricità con miglioramenti dell'umore») (Proietti, La Gatta, 2005, p.7); contrastando la sedentarietà e l'attività cardio-circolatoria, nonché amplificando in senso positivo lo stato emotivo e psicomotorio (Ballarini, 2000) ${ }^{5}$.

Analogamente, nelle famiglie a genitore unico o monoparentali - caratterizzate dalla presenza di un'unica figura genitoriale che si prende cura della prole in fase di post-separazione coniugale o per una condizione di vedovanza o come altra forma di convivenza - la familiarità ed il contatto corporeo con un animale da compagnia attivano un insieme strategie di enrichment familiare: svolgendo una funzione pseudo-compensativa, rispetto alla possibilità di spostare la propria attenzione da una relazione mancante ed assente ad una nuova e presente; potenziando competenze (ad esempio, sul registro genitoriale, nel caso di tale tipologia familiare) verso la risoluzione di blocchi emotivi, che affiorano nei delicati momenti di passaggio della vita individuale e familiare. Il focus di tali strategie potrà ulteriormente spostarsi verso i processi di resilience, finalizzati ad interventi di promozione delle risorse e del benessere familiare (ad esempio, di sostegno alla genitorialità).

Nei nuclei familiari ricomposti, in cui il Pet interagisce con tutti i membri familiari acquisiti nella nuova ricomposizione familiare, i processi interattivi possono essere e vengono veicolati da

4 Con il termine anglosassone pet si fa riferimento all'Animale domestico o familiare. In particolare, i concetti scientifici intorno ai pets si sono estesi oltre che ai cani e ai gatti, anche a tartarughe e pesci rossi che vivono in casa.

5 Una ricerca condotta su un gruppo di famiglie unipersonali (48 soggetti di età compresa dai 75 agli 81 anni, residenti nel Regno Unito a cui erano stati affidati dei pappagalli, piante da curare o un elettrodomestico), ha evidenziato un effetto positivo sul benessere psicofisico solo nel gruppo di soggetti che hanno vissuto in presenza di animali domestici (in Ballarini, 2000, p. 19). 
forme linguistiche semplici e da espressioni che ne valorizzano la cadenza, il tono, l'inflessione, lo sguardo, con un effetto rilassante e rassicurante su tutti i componenti (Proietti, La Gatta, 2005). Mediante un approccio proattivo, in altri termini, tali processi hanno in sé le potenzialità di sciogliere nodi problematici ed aprire uno scenario in cui diventerà possibile porre attenzione alle dinamiche relazionali della famiglia, riconoscerne gli scambi affettivi, i significati ed i valori.

Le Pet Relationships si configurano in tal senso nei contesti familiari con funzioni molteplici, in grado di impedire o alleviare stati emotivi negativi, facilitare strategie di coping e di empowerment per il superamento di eventi critici, individuali o familiari, normativi e non.

Tra le diverse forme di sostegno mirate al buon funzionamento familiare, la Zooantropologia favorisce particolarmente l'attivazione di meccanismi adattivi. Quale attuale approccio di studio e di ricerca, la Zooantropologia Didattica ${ }^{6}$ si configura come un sistema di mediazione uomo-animale che avvia processi d'interazione con significativi risvolti riabilitativi e psicoeducativi, in diversi ambiti applicativi. Viene pertanto focalizzato il processo di interscambio nel sistema di mediazione uomo-animale, basato sul valore della relazione, sul ruolo dialogico dell'eterospecifico e sulla riconsiderazione dell'animale d'affezione non più reificato, né antropomorfizzato (Marchesini, Corona, 2007; Marchesini,2007). In altri termini, l'accento sul valore dell'alterità consente di rintracciare le basi di una particolare grammatica emotiva, il cui potenziale consiste proprio nella diversità specie-specifica che può svelarsi anche in un setting di cura (come si descriverà più avanti).

Più dettagliatamente, strutturando specifici contesti ed attività assistite, tale approccio consente lo sviluppo di processi e competenze emotive e sociali (quali: il pianificare, il relazionarsi, il comunicare), necessarie per l'attraversamento del rischio individuale

${ }^{6}$ Per ulteriori approfondimenti sui presupposti dell'approccio zooantropologico si rimanda a: Montagner H.(2001).Il bambino, l'animale, la scuola. Bologna: Oasi Alberto Perdisa; Marchesini R. (2005).Fondamenti di Zooantropologia Applicata. Bologna: Oasi Alberto Perdisa. 
e familiare(Giusti, La Fata, 2004). In particolare la Pet therapy $^{7}$, tra le attività terapeutiche assistite in co-presenza di Operatore-AnimaleUtente/i, ha come obiettivo d'intervento non il partner (umano e animale) preso singolarmente ma il rapporto uomo-animale come realtà emergente, capace di liberare nei membri di una famiglia - nel bambino, nell'anziano, nel disabile - risorse interiori che ordinariamente vengono represse nell'ambito dell'interazione interumana (Marchesini, 2005).

Da un punto di vista psicoeducativo, infatti, tramite l'interazione bambino-animale si struttura, attraverso delle Attività di Pet Relationship (APR), una serie di occasioni di referenza educativa (Pet Pedagogy) con specifiche competenze formative: la capacità di aumentare il vocabolario immaginativo e le abilità comunicative, di diminuire la diffidenza verso la diversità, amplificando invece la capacità empatica, la capacità di problem solving per il superamento delle fasi di passaggio più critiche. Ulteriori applicazioni in ambito zooantropologico offrono peraltro linee-guida di didattica ambientale, favorendo un atteggiamento educativo all'incontro con l'animale, teso a fugare nel bambino anche situazioni interattive psicopatologiche: zoofobie, zoointolleranze, zoo manie, etc. (ibidem,2005).

Numerosi sono inoltre gli studi che valorizzano l'ambito applicativo della pet therapy nel contesto ospedaliero e, in particolare, in quello pediatrico.

Le modalità di presa in carico animal assisted (Odendall, 2000), si orientano verso la riduzione delle potenzialità di un trauma psicologico in un bambino ospedalizzato agendo, in un'ottica sistemico-relazionale attenta alla malattia come un evento che altera il ciclo vitale e il corpo familiare (Cigoli, 1995; Andolfi, Mascellani, 2010), sui sistemi di riferimento coinvolti (famiglia, personale sanitario e parasanitario dell'ospedale). Mediante attività in aree

\footnotetext{
${ }^{7}$ Il termine Pet Therapy indica l'utilizzo della relazione uomo-animale in campo medico e psicologico per apportare benefici di ordine terapeutico ed assistenziale, valorizzando proprio l'aspetto terapeutico insito nel rapporto con l'animale che entra in contatto con il vissuto umano (del bambino, dell'adolescente, etc). Per maggiori approfondimenti si rinvia a: Del Negro E. (2004). Pet Therapy. Una proposta d'intervento per i disabili neuromotori e sensoriali. Milano: FrancoAngeli.
} 
opportunamente individuate del contesto ospedaliero, lo spazio ed il tempo dell'ospedalizzazione possono iniziare a connotarsi come esperienze di contatto emozionale e corporeo, contrastando la staticità e la monocromaticità della malattia e della degenza ospedaliera, per focalizzarsi sul "qui ed ora", quale dimensione temporale privilegiata per stabilire una relazione d'aiuto con un bambino ospedalizzato (Fine, 2000).

Alla luce di tali presupposti, attraverso la pet pedagogy il rapporto con l'animale familiare entra nel vissuto del bambino o dell'adolescente per facilitarne la crescita, lo sviluppo psichico e morale diventando una forma di sostegno nella complessità delle situazioni e nella quotidianità (Levinson,1982). In tal senso, diviene possibile rintracciare una specifica valenza riparativa di tale approccio, ovvero, una particolare "forza educativa" insita negli animali domestici (Bettetini, 2006), delineabile anche negli ambienti familiari caratterizzati dalla presenza di un evento critico non normativo, ovvero, non scelto ed inatteso. Ad esempio, prendendo in considerazione la capacità di adattamento della famiglia in seguito alla nascita di un bambino disabile, in rapporto alla qualità d'impegno nella cura, alle strategie di coping messe in atto, le APR possono inserirsi tra i diversi livelli della rete di supporto. Spesso, infatti, le famiglie messe di fronte all'evento paranormativo della disabilità faticano ad interagire con il tessuto sociale ed a gestire i vissuti connessi con la malattia stessa. Nel pieno rispetto dei tempi e delle caratteristiche proprie e dell'utente, gli obiettivi delle attività PR si orienteranno innanzitutto sul piano di una stimolazione multisensoriale attiva o passiva (motoria, comunicativa, etc.) del bambino che vive in condizioni di disabilità. In particolare, sul piano del contatto corporeo - ove la dimensione corporea prevale certamente su quella linguistica - un pet è in grado di esprimere in modo coerente le proprie emozioni tramite la mimica del corpo ed i suoni vocali, utilizzando posture che favoriscono la percezione corporea; nelle attività assististe operatore-pet-utente è possibile generare un rilassamento muscolare ed uno stato generale di benessere utili non soltanto per il soggetto che vive in una condizione di handicap ma amplificabile all'intero sistema familiare, attivando 
una comunicazione intercorporea portatrice di una difficoltà proattiva che sollecita un nuovo adattamento familiare (Del Negro, 2004).

Entro tali delicate e complesse dinamiche familiari, la presenza di un pet può svolgere una funzione di "ammortizzatore" degli stress emotivi, ove l'entrata di un cucciolo in un ambiente familiare caratterizzato da tali blocchi nel ciclo evolutivo può rappresentare il tentativo di superare una dimensione di "congelamento" del corpo e della mente (Hutton, 1983).

\section{Pet relationships: ambiti di studio, questioni metodologiche e contesti di cura}

«Relationships between people and animals have been ignored by social researchers for a long time. The main reason for this has been an assumption that achievement of intersubjectivity in testifying of animals' inner experiences and feelings is impossible. All "reactions" of animals to our stimuluses have been treated as one side interpretation due only to human actor» (Konecki, 2007, p.2).

Negli ultimi vent'anni, un vasto numero di ricerche relative ai benefici della relazione tra esseri umani ed animali da compagnia si è posto l'obiettivo di indagare sulle questioni metodologiche ad essa circoscritte, evitandone il rischio di una frammentazione in un campo così ampiamente attraversato da diverse discipline (Wilson, 1998). Differenti ma proficuamente incrociabili risultano gli approcci di studio di tipo qualitativo finora maggiormente utilizzati: dall'interazionismo-simbolico post-meadiano, all'etnometodologia degli analisti, al post-umanesimo dei sociologici. In particolare, uno dei principali problemi sollevati riguarda lo status dell'animale coinvolto: what is the nature of animal action and interaction as it become available to the qualitative researcher? 
Nonostante l'influenza cartesiana abbia ridotto l'agire animale ad una serie di meccanismi istintivi, nonché di comportamenti precostituiti, altri studi sottolineano invece i benefici degli animali da compagnia per il benessere degli esseri umani evidenziando in particolare l'importanza della loro relazione e la modalità con cui essa può evolversi (Travers, 2001). Se essa rappresentasse il mero risultato di meccanismi istintivi, non si spiegherebbe infatti la sua evoluzione: recenti dati, focalizzati sul rapporto tra cani e gatti ed i loro padroni, ne dimostrano le modalità evolutive e distintive (Haraway 2003; Bekoff et al., 2002); e se questa risultasse la questione centrale da ampliare, la ricerca qualitativa offrirebbe la possibilità di esplorarne la natura, i suoi mutamenti ed i suoi benefici, andando oltre i dati statistici.

Un ulteriore aspetto riguarda peraltro la posizione di "secondo posto" assegnata alla ricerca in tale ambito che invece offrirebbe nuove aperture, attraversando l'annoso dibattito filosofico tra scienze umane e scienze esatte (Myers,2003).

Una questione particolarmente discussa è inoltre quella relativa al legame tra mondo animale ed umano: alcuni studi sociologici esplorano attualmente la dimensione intersoggettiva nell'interazione uomo-animale, osservabile mediante un approccio fenomenologico che configura la personalità e la personalità animale, come intersoggettivamente connesse (Taylor, 2004; 2007). In realtà, la sociologia ha finora negato la possibilità che l'interazione umana $\mathrm{e}$ quella con altri animali non umani possa essere considerata di tipo sociale: sociology as if nature did not matter (Murphy, 1995, p.689). Tale principio si basa da un lato sull'assunto post-cartesiano che nega una dimensione intercorporea, affermando invece la distinzione tra mondo oggettivo e soggettivo, e dall'altro rispetto ad una corrente sociologica post-illuminista che vede il Sé come risultato dell'ascesa dell'uomo dall'animalità («itself in terms of man's ascent from animality ») (Murphy ibidem, p. 689) e che consolida il punto di vista antropocentrico del mondo, connotando allo stesso tempo le interazioni sociali nei termini di «unidirectional causality from the social to the natural» (Murphy, ibidem, p.690). Tale mancanza di interesse per l'interazione uomo-animale trova peraltro le sue 
premesse nell'affermazione di George Mead (1934) ${ }^{8}$, per cui l'interazione simbolica avviene solo quando chi interagisce possiede un senso del Sé, che appartiene solo agli esseri umani. In altri termini, per Mead soltanto gli esseri umani possono essere considerati in grado di creare interazioni sociali, per la loro competenza linguistica e simbolica che ha la funzione di interpretare i gesti intenzionali degli altri (Alger \& Alger, 1997). Diversamente, Collins (1989) rifiuta tale assunto, affermando la distinzione tra interazioni tra esseri umani e gli altri animali non umani. Durante l'ultima decade affiorano diversi tentativi di cambiamento di tale prospettiva antropocentrica: Sanders (2003), insieme ad altri studiosi (Arluke 2003; Alger \& Alger 1997; 2003; Myers 2003), afferma la non legittimità dello studio della natura di tale interazione in ambito sociologico, per collocarla piuttosto entro un approccio di studio fenomenologico e etno-metodologico che configura la mente come una costruzione sociale piuttosto che come un dato meramente biologico, ove il mondo sociale rappresenterebbe l'esito di un'esperienza intersoggettiva (Coulter 1989). Tale approccio, che vede un mondo intersoggettivamente costruito, include anche la presenza degli animali all'interno della cultura umana aprendo alla possibilità di potere studiare il loro ruolo nella società attraverso l'interazione uomo-animale: l'agire con gli animali è concepito come un incontro tra due mentalità interagenti (Taylor, 2007). Come spiega ancora Krzysztof (2007), uno degli strumenti preferenziali per osservare da un punto fenomenologico l'intersoggettività rimane l'azione, collocabile entro un contesto temporale del qui e ora che riconosce la co-presenza di due corpi interagenti: il linguaggio simbolico è soltanto uno degli elementi dell'azione ma l'assumere il ruolo dell'altro consente di avviare un contesto intersoggettivo tramite un agire non verbale (o non necessariamente linguistico) diretto ai partners dell'interazione. Ad esempio, se un cane reagisce all'invito di giocare, risulta difficile negare la sua comprensione delle regole del gioco o dell'attività che socialmente definiamo "gioco". Tali regole vengono apprese attraverso uno scambio di azioni (gesti),

\footnotetext{
${ }^{8}$ Mead G.H. (1934) Mind, Self, and Society. Chicago: University of Chicago Press.
} 
che assumono gli stessi (o simili) significati per entrambi i partner dell'interazione (Taylor, 2007).

Sanders $(1993,2003)$ specificatamente approfondisce tale natura intersoggettiva, esplorando la modalità con cui una pet relationship modella l'identità del caregiver umano (ad esempio del padrone $\mathrm{o}$ dell'operatore socio-sanitario); in particolare, nelle sue ricerche sulla percezione degli operatori che lavorano con soggetti che vivono in condizioni di grave disabilità, Sanders evidenzia una differenza percettiva significativa tra gli operatori che hanno un animale domestico e che percepiscono i soggetti disabili come esseri umani a pieno titolo, rispetto agli altri operatori, privi invece di esperienze di pet relationships, che mostrano difficoltà nell'individuare gli utenti come "persone" a tutti gli effetti. Tali dati sottolineano l'elemento intersoggettivo, rintracciabile attraverso le quotidiane e strette interazioni tra gli operatori ed i propri cani, che faciliterebbero l'apprendimento del senso dell'unicità di ciascuno essere vivente, una spiccata capacità empatica ed altresì la condivisione dei ruoli sociali, nonostante l'assenza di un linguaggio verbale.

Nell'intento di esplorare le caratteristiche di un'esperienza di pet relationship in ambito psicologico e clinico, non è infrequente incontrare nella storia della psicoterapia diversi terapeuti che hanno avuto un rapporto significativo con un cane o con altri animali. In particolare Francesco Bruni, coinvolgendo alcuni psicoterapeuti (di orientamento diverso: psicoanalisti, freudiani e junghiani, cognitivisti e sistemico-relazionali), porta alla luce suggestive storie di vita che li vedono coinvolti con i propri animali, sia dentro che fuori la stanza di terapia, evidenziando come tale presenza possa contribuire ad acuire la sensibilità clinica e la capacità empatica del terapeuta. Alla luce dell'etologia e della teoria dell'attaccamento (Howes, 1999) e prendendo in esame la comunicazione uomo-cane, nonché le esperienze emotive che l'accompagnano, Bruni (2009) avvalora soprattutto la presenza, reale e simbolica, degli animali nella vita quotidiana dello psicoterapeuta nel suo lavoro ed altresì estende la rilevanza di tale ambito di indagine dell'incontro uomoanimale allo studio dello sviluppo del bambino, all'età adulta ed al sistema familiare: « Non ero stato allevato in campagna e non avevo 
avuto animali. Da piccolo avevo vissuto con qualche pesce rosso e mi ero sempre chiesto se a loro piacesse stare nell'acquario. Ma in casa mia ho sempre percepito molto amore e compassione e so che questo è collegato con il mio amore per gli animali ») (Bekoff, 2002, p.39).

Come ricorda Marshall Thomas, l'animale d'affezione assume un ruolo significativo tra $\mathrm{i}$ caregivers significativi in quanto presenza familiare e semplicemente in quanto presenza senziente, dotata di una vita emotiva complessa e degna di rispetto (Pet Care $)^{9}$, che mobilita una serie di fattori di facilitazione delle interazioni interpersonali con risvolti educativi: «Quando ero molto piccola, la mia tata era un'enorme cagna terranova chiamata Mishka, che aveva il compito d'impedirmi di annegare. A quell'epoca la nostra famiglia andava in vacanza a Cape Cod e Mishka che, al pari di molti cani, conosceva ogni sfumatura del comando "Seduta e ferma", mi costringeva a fare sulla spiaggia esattamente questo: restare seduta e ferma a distanza notevole dall'acqua. Trascorrevo così molte giornate estive, madida di sudore e con la bocca arida, osservando delusa gli altri che sguazzavano felicemente in acqua, perché la vecchia e irascibile Mishka, la cui severità superava di molto quella dei membri adulti della mia famiglia, non mi permetteva neppure di raggiungere la riva per costruirvi un castello di sabbia») (Marshall Thomas, 2000, p.9).

\section{La storia di Lucy e Fey come incontro eterospecifico e terapeutico $^{10}$}

\footnotetext{
${ }^{9}$ Per approfondimenti sulla Pet Care e sulla Pet Pedagogy si rinvia a: Levinson B.M.(1982).Pets and Human Development. Springfield: Charles Thomas; Marchesini R.(2011). Modelli cognitivi e comportamento animale. Coordinate d'interpretazione e protocolli applicativi. Venafro(IS):EvaEdizioni; Bettetini M.(2006). Amici Pelosi e altre bestie. La forza educativa degli animali. Cinisello Balsamo: San Paolo.

10 Si precisa che l'esemplificazione clinica riportata riguarda il trattamento individuale, nonostante le premesse teoriche abbiano come sfondo il significato della presenza dell'animale all'interno della famiglia, poiché in questa specifica vignetta non si è potuta prendere in considerazione una presa in carico di tipo familiare.
} 
Lucy viene nel mio studio all'età di 16 anni, su richiesta della sua famiglia: prima di addormentarsi deve portarsi il dito in bocca, ciucciarlo per tutta la notte e contemporaneamente iniziare a tirare $\mathrm{i}$ suoi capelli talmente forte, fino a strapparli.

Dopo alcune settimane di terapia, in cui emerge inizialmente anche una fobia particolare verso le piume dei volatili (dei piccioni, in particolare), l'atteggiamento di chiusura ed il vissuto di costrizione nei confronti della sua famiglia (e certamente anche verso di me), sembrano non trovar tregua in Lucy. Le nostre sedute proseguono ma sono caratterizzate da lunghi momenti di silenzio, in apparenza privi di significato, da interruzioni repentine con le quali Lucy chiede di finire prima del previsto l'incontro, da improvvisi impegni di dopo scuola che le consentono di rinviarle di settimana in settimana. Questo, il clima che caratterizza i nostri incontri per un paio di mesi. Fino al giorno in cui, dal vetro della porta della stanza del mio studio - involontariamente - Lucy e Fey ${ }^{11}$ s'intravedono. Per la prima volta, L. si rivolge a me con una richiesta: "Per favore, puoi fare entrare il tuo cane nella stanza?"

Da quell'incontro relazionale, imprevisto ma propizio, il setting comincia a svelarsi nelle sue caratteristiche, attivando un processo terapeutico ed assumendo le funzioni di una co-terapia unica nella sua efficacia: proprio la natura molossoide ${ }^{12} \mathrm{di} F e y$, che la porta a richiedere costantemente un contatto corporeo (anche semplicemente ponendo la zampa sul piede di L. o appoggiandole il muso sulle gambe), attiva un contesto di "co-creazione" tra un graduale

\footnotetext{
11 Fey è il nome della mia Rottweiler. Sin da cucciola - già a 2 mesi d'età - mi accompagna nella vita affettiva e anche professionale. Durante le sedute, Fey decide di rimanere libera in una parte dello studio o, come nel caso sopradescritto, di entrare con me nella stanza di terapia.

12 Il termine fa riferimento alla famiglia di razze canine (Molossi), caratterizzati da un carattere sensibile e deciso, una particolare robustezza fisica ed un forte attaccamento al padrone, al punto da sentirne il bisogno di uno stretto contatto fisico. Il molosso ha infatti la necessità di un compagno che lo indirizzi in maniera corretta e semplice , con una educazione mai basata sulle punizioni bensì sulle attività esplorative e ricreative.
} 
contattarsi corporeo, con manipolazioni ed attività esplorative, fino a sbloccare la spontaneità dell'intenzionalità di contatto ${ }^{13} \mathrm{di}$ L.

In termini psicodinamici, Fey aiuta L. - seduta dopo seduta - ad esplicitare i suoi vissuti emotivi e corporei: innanzitutto, la sua tristezza: che emerge e si esprime attraverso le lacrime prontamente asciugate dalla lingua di Fey; la sua rabbia: che inizia - finalmente a canalizzarsi in energia manipolativa verso l'ambiente, attraverso ripetute attività di "tira e molla" con il manicotto" ${ }^{14}$; ancora, la sua solitudine: che lascia posto alla presenza e al calore, alla spontaneità e alla leggerezza, stimolata dai puntuali ed "esatti" richiami effettuati da Fey, tramite la sua zampa.

Come unica mediatrice, mi sento un'osservatrice in una corsia preferenziale ma allo stesso tempo inizio anche io a rendere attivo un setting di coterapia, già avviato dalla mia copartner. Il processo terapeutico si snoda e consente innanzitutto al non detto di diventare detto, rendendo esplicito il significato relazionale dei vissuti di L., nei confronti di chi, finora, non è stato un caregiver adeguato nel suo ambiente primario.

Mediante la dimensione dell'intercorporeità ${ }^{15}$, che guarda all'incontro dei corpi come il luogo in cui vive l'identità relazionale, si apre un lavoro sulla consapevolezza tra i nostri corpi, nel setting di coterapia.

\footnotetext{
13 Per ulteriori approfondimenti sul concetto di Intenzionalità di contatto secondo la prospettiva della Gestalt Therapy si rinvia a: Salonia G. (2001). Tempo e relazione. L'intenzionalità relazionale come orizzonte ermeneutico della Psicoterapia della Gestalt In: Spagnuolo Lobb M. (a cura di), Psicoterapia della Gestalt. Ermenentica e clinica. Milano: Franco Angeli, pp 65-85; Spagnuolo Lobb M.(2011).Il now for next in psicoterapia. Milano: Franco Angeli

${ }^{14}$ Il gioco del manicotto (o del "tira e molla") costituisce un'attività ludica molto stimolante. In particolare, il manicotto consente di aumentare l'istinto predatorio e possessivo del cane. Attraverso questo oggetto, solitamente composto da una tela di iuta, si attiva il gioco del "tira alla fune" che, come momento di distensione e destressamento, consente al cane e al padrone di rafforzare la loro intesa.

${ }^{15}$ Sul concetto di intercorporeità in PdG si rinvia a : Salonia G., Edipo dopo Freud.Gestalt Therapy e teorie evolutive. Trapani: Il Pozzo di Giacobbe, 2013; Merleau-Ponty M. (1979), Il corpo vissuto, a cura di F. Fergnani. Milano: Il Saggiatore; Salonia G. (2010). L'anxiety come interruzione nella Gestalt Therapy In: Regazzo L.D. a cura di, Ansia, che fare? Prevenzione, farmacoterapia e psicoterapia. Padova: CLEUP.
} 
Dal punto di vista metodologico, ad esempio, l'avvicinare (nella fantasia) l'oggetto fobico al paziente, ha lo scopo di far prendere consapevolezza del vissuto corporeo e relazionale che l'oggetto stesso evoca. Il passaggio dall'oggetto fobico al vissuto permette in altri termini al corpo del paziente - sostenuto dal corpo e dalla relazione del terapeuta - di consapevolizzare e riuscire a contenere l'eccitazione e l'energia che evita. In particolare, con gli adolescenti si rivela molto utile la metafora dell'accostarsi all'oggetto fobico con una "bacchetta magica", metafora della forza e del potere che l'Organismo sperimenta con difficoltà (Salonia, 2011).

Analogamente, quando chiedo a L. di immaginarsi in presenza del suo oggetto fobico (i piccioni, con le loro piume), usando la bacchetta magica e scegliendo qualcuno nella sua vita attuale che possa starle vicino per darle sostegno, lei non esita a ricorrere a Fey! La chiama a sé ed inizia a lasciarsi andare, sperimentando la sensazione di ricevere calore, nel e dal suo corpo. Gradualmente, si ripristina la fiducia di base attraverso un fidarsi corporeo - tra L. e Fey- e un dare significato relazionale all'esperienza, in un ambiente triadico in cui L. è l'elemento terzo che attiva un coppia cogenitoriale (o coterapeutica, formata da me e Fey) .

Riguardo alle fobie di animali (come fobie monotematiche), secondo la prospettiva della psicoterapia della Gestalt, può inoltre risultare funzionale chiedere alla persona di identificarsi con l'animale di cui si ha paura, compiendone i gesti tipici, poiché spesso, proprio nella descrizione dell'oggetto fobico, il paziente esprime i vissuti di cui ha paura: «la fobia è fobia di ciò che non faccio, non esprimo» (ibidem, 2011, p.47). In realtà, infatti, L. non ha di per sé paura dei piccioni ma delle sensazioni provocate dal loro sbattere le ali o dallo sfregamento su di sé (descritto come irritante e disgustoso) delle loro piume.

Assecondando questa modalità, L. inizia a muovere le braccia, quasi imitando il battere delle ali, in un volo che le dà respiro e la prepara a lasciarsi andare al fluire delle sue emozioni incompiute e non adeguatamente sostenute dall'ambiente primario. I pensieri disfunzionali, originati da un'interruzione di un processo respiratorio 
nel suo senso corporeo-relazionale, diventano adesso pensieri liberi dai vissuti di paura.

Rispetto invece alla modalità (di tipo ossessivo-compulsiva) del succhiare il dito prima di addormentarsi, il setting rievoca il ricordo della paura non contenuta dall'ambiente primario e anzi trasformata in terrore. Per F. Perls i pensieri ossessivi rappresentano proprio un succhiotto che: «(...)permette lo scarico di una certa dose di aggressività, ma non produce nessun cambiamento nel bambino, cioè non lo nutre $>$ (Perls, 1995, pp.146-47).

Proprio mediante tali pensieri e modalità, disfunzionali e dolorose, L. ha potuto prendersi cura di se stessa con una forma di attaccarsi (a se stessa) che evita il rischio di un cambiamento nelle relazioni, bloccando la paura della separazione che è nel suo sfondo ed esercitando un eccessivo controllo della sua aggressività, vissuta come un'energia emozionale che la terrorizza.

L'assenza o l'interruzione di cura da parte delle figure genitoriali sembra avere generato una difficoltà nel controllo spontaneo dei propri vissuti: dopo avere ricevuto il primo sostegno, L. da primogenita è stata infatti "messa da parte" alla nascita della sorella, avvenuta quando lei compie il terzo anno di età. Come se, dopo avere ricevuto il primo sostegno nel sentire $\mathrm{i}$ vissuti, successivamente a quell'evento le sia mancato quel sostegno specifico nel lasciarsi andare fino in fondo al fluire delle sue emozioni. E, come afferma Salonia (ibidem, 2001, p.36): «l'assenza di sostegno se non trova soluzione, lascia il posto ad un'angoscia di morte (morte propria, ma anche morte delle persone care indispensabili), che travolge». La paura di fondo di L. è proprio quella del separarsi e dell'avere emozioni proprie, con il rischio di essere messa da parte, ancora una volta. Il vissuto corporeo relazionale che L. tiene segreto è il terrore: di sentire l'energia attivata nel corpo, dell'azione che porta all'emozione, di separarsi e trasgredire.

Dare sostegno al suo corpo impaurito (come riaprire la possibilità di una respirazione che scorre spontanea verso il compimento dell'intenzionalità di relazione;cfr Salonia, 2008) è stato allora innanzitutto il primo passo coterapeutico. Attraverso il lavoro corporeo, si è attivato un percorso di consapevolezza, come capacità 
di identificarsi con un'intenzione non chiara e non espressa. Il gesto dello strapparsi i capelli, simile ad una compulsione di contenimento, pare rivelare l'intensificarsi della paura di non sapere controllare un'intenzione. In tal senso, l'azione compulsiva di L. blocca la sua spontaneità e ha la funzione di accrescere il controllo su quelle emozioni percepite come distruttive.

Quando chiedo a L. di ripetere il gesto del tirare i capelli, che automaticamente attiva quello del mettersi il dito in bocca, la sua tensione muscolare, il suo sguardo impaurito che cerca conforto, lasciano spazio ad un gemito che Fey immediatamente avverte e contiene, poggiando il muso sulla bocca di L. fino al toglierle la mano dalla testa, già serrata ai capelli. Rimanendo alle sue spalle, F. inizia inoltre a leccarle le lacrime, che lentamente stanno scivolando sul viso di L., ed a mitigarne i singhiozzi. Dopo un lungo abbraccio (nella triade), finalmente il corpo di L. sente la sua energia e, iniziando a fidarsi dell'ambiente sperimentato in seduta, non tenta più di tenerla sotto controllo. Il suo schema corporeo, prima quasi rimpicciolito, si apre alla novità, ad un nuovo corpo che le consente il "lasciarsi andare con", toccando un corpo adesso vissuto".

\section{Risvolti psicoeducativi e terapeutici}

\footnotetext{
16 Secondo la prospettiva della Gestal Therapy, risulta importante distinguere il corpo "visto" da quello "vissuto"; mentre il primo rientra nel criterio valutativo, per cui il corpo viene immaginato in base a ciò che si vede (ad esempio: la mia mano non mi piace perché è grande!) o in base alla sua funzionalità (ad esempio: un corpo che danza, dove quando il corpo si muove lo si immagina in funzione di un movimento), il corpo vissuto (o abitato) fa riferimento ad un aspetto puramente fenomenologico in cui il concetto di bellezza è legato al modo di percepire il proprio corpo (ad esempio: sento la mia mano morbida; se ho questa percezione della mano, la vedo e so anche come si muove). Per ulteriori approfondimenti si rinvia a: Salonia G. (2008), La psicoterapia della Gestalt e il lavoro sul corpo. Per una rilettura del fitness. In: Vero S., Il corpo disabitato. Semiologia, fenomenologia e psicopatologia del fitness. Milano:Franco Angeli.
} 
« Future human-animal investigations should probably focus less on unverifiable speculations about the inner lives of animals and examine instead what is knowable about human-animal interactions and the significance that humans attribute to them») (Jerolmack, 2005, p. 660).

Al di là di un moda zooantrolopologica, ogni animale per le sue valenze terapeutiche ed educative merita maggiore dignità: non come strumento ma come «presenza che s'inserisce nell'insieme di una famiglia, fino a modificarne la dinamica transgenerazionale (Andreoli, 2004, p.153)».

Tenendo conto dei presupposti della Zooantropologia Didattica, che come attuale approccio di studio e di ricerca individua nel sistema di mediazione uomo-animale un processo di interscambio basato sul valore della relazione evitando applicazioni antropocentriche, è nell'incontro eterospecifico che è possibile delineare significativi risvolti psicoeducativi e terapeutici delle applicazioni di tale approccio in diversi ambiti. Sulla base di tali presupposti, fare riferimento alla prospettiva zooantropologica durante il ciclo vitale consente da un lato di leggere da un punto di visto diacronico la storia dell'individuo e del suo ambiente familiare, dall'altro nei termini di una epistemologia relazionale può e dà spazio al modo in cui organismo e ambiente s'incontrano e si autoregolano in un "tra" unico ed irripetibile, da cui può emergere una realtà nuova, co-creata e non data dalla somma delle parti. Tale prospettiva introduce, in altri termini, ad una grammatica emotiva eterospecifica, ove si apprende un modello d'interazione con un essere diverso da sé, in cui l'altro diviene il Tu da scoprire e l'Io accetta di accogliere e donare nella relazione. Tale dinamica può favorire una transizione tra l'Io e il Tu, anche come elemento di dialogo transgenerazionale (Pagani, 1999; 2004).

Ulteriori riflessioni si orientano sul versante epistemologico ed etico, di un adeguato modello e di un valido metodo scientifico: esiste davvero una buona scienza che tenga conto delle peculiarità e del benessere psicofisico ed emotivo di coloro che ne vengono coinvolti? 
Ovvero: quale dimensione socio-culturale può nutrire una mentalità zooantropologica che tenga conto del valore relazionale?

Come afferma Mark Bekoff è una buona regola riconciliare il senso comune, con il senso della scienza: «Gli animali, nostri parenti, dipendono dalla nostra buona disposizione d'animo e dalla nostra clemenza. Gli animali dipendono da ciò che gli esseri umani hanno in mente rispetto a quali sono i loro migliori interessi. La scelta è tra intromettersi, abusare ed essere compassionevoli. Non dobbiamo fare qualcosa soltanto perché possiamo farla. Ognuno di noi è responsabile delle proprie scelte, che dovrebbero essere basate su alcuni ideali: 1) il rispetto, la compassione e l'ammirazione per gli altri animali; 2) porsi seriamente dal loro punto di vista; 3 ) stare dalla parte degli animali rischiando di peccare per eccesso quando siamo incerti riguardo alla loro sofferenza(...)〉(Bekoff, 2002, p. 69).

\section{Riferimenti Bibliografici}

Alger J. \& Alger S. (1997). Beyond Mead: Symbolic interaction between humans and felines. Society and Animals 5(1): 65-81.

Alger J. \& Alger S. (2003). Cat culture : the social world of a cat. Shelter. Philadelphia: Temple University Press.

Andolfi M. \& Mascellani A. (2010). Storie di adolescenza. Esperienze di terapia familiare. Milano: Cortina

Andreoli V.(2004). Istruzioni per essere normali. Milano:Bur

Ballarini G. (2000). Animali: terapia dell'anima. Brescia: Fondazione iniziative zooprofilattiche e zootecniche.

Bekoff M.(2002). Dalla parte degli animali. Roma: Muzzio.

Bekoff M., Colin A. \& Gordon B.(2002). The cognitive animal: empirical and theoretical perspectives on animal cognition. Cambridge, Mass: MIT Press.

Bettetini M.(2006). Amici Pelosi e altre bestie. La forza educativa degli animali. Cinisello Balsamo: San Paolo.

Bruni F. (2009). Lo psicoterapeuta ed il cane. Emozione, comunicazione, relazione. Torino: Antigone.

Cigoli V.(1995). Il corpo familiare. Milano: FrancoAngeli. 
Collins R. (1989). Toward a neo-Meadian sociology of mind. Symbolic Interaction 12(1): 1-32.

Coulter J.(1989). Mind in action. Cambridge: Polity Press.

Del Negro E.(2004). Pet Therapy. Una proposta d'intervento per $i$ disabili neuromotori e sensoriali. Milano:FrancoAngeli.

Giusti E., La Fata S. (2004).Quando il mio terapeuta è un cane. Roma:Sovera

Haraway D.(2003). The companion species manifesto. Chicago: Prickly Paradigm Press.

Hutton J.S. (1983). The human- pet relationships, In: Animali: terapia dell'anima. Brescia: Fondazione iniziative zooprofilattiche e zootecniche.

Howes C. (1999). La relazione di attaccamento nel contesto di caregiver multipli (pp. 761-779). In: Cassidy J.\& Shaver C., a cura di, Manuale dell'attaccamento. Roma:Fioriti.

Jerolmack C.(2005). Our animals, our selves? Chipping away the human-animal divide. Sociological Forum 20:651-660.

Konecki K.T. (2007). Editorial: People and Animals. On the problem of intersubjectivity in interactions of humans and animals, Qualitative sociology Review, 3, 1: 3-5.

Levinson B.M.(1982). Pets and Human Development. Springfield: Charles Thomas.

Marchesini R. \& Corona L.(2007). Attività e terapie assistite dagli animali. L'approccio zooantropologico alla pet therapy. Bologna: Oasi Alberto Perdisa.

Marchesini R. (2005).Fondamenti di Zooantropologia Applicata. Bologna: Oasi Alberto Perdisa.

- (2007). Pedagogia cinofila. Introduzione all'approccio cognitivo zoo antropologico. Bologna: Oasi Alberto Perdisa.

Marshall Thomas E.(2000). La vita sociale dei cani. Milano:Tea.

Murphy R.(1995). Sociology as if nature did not matter: an ecological critique. British Journal of Sociology 46(4): 688707. 
Myers O. E. (2003). No longer the lonely species: a post-Mead perspective on animals and sociology. International Journal of Sociology and Social Policy 23(3): 46-68.

Netting F.E., Wilson D.D.\& New JC.(1987) The human-animal bond: implications for practice. The British Journal of Social Work.32:60-64

Odendall J.S.J.(2000).Animal assisted therapy: magic or medicine? Journal of Psychosomatic Research,49:275-280.

Pagani C.(1999).Bambini che maltrattano gli animali. Psicologia contemporanea. 153: 30-37.

Pagani C.(2004). Pet in famiglia. Impronte.9: 32-33.

Perls F. (1995). L'io, la fame e l'aggressività.Milano: Franco Angeli

Proietti G.\& La Gatta W.(2005). La Pet Therapy. Milano: Xenia

Salerno A.(2010).Vivere insieme. Tendenze e trasformazioni della coppia moderna. Bologna: Il Mulino

Salonia G. (2008). La psicoterapia della Gestalt e il lavoro sul corpo. Per una rilettura del fitness. In: Vero S., Il corpo disabitato. Semiologia, fenomenologia e psicopatologia del fitness. Milano: Franco Angeli.

Salonia G.(2011). L'angoscia dell'agire tra eccitazione e trasgressione, La Gestalt Therapy con gli stili relazionali fobico, ossessivi compulsivi, GTK, Rivista di Psicoterapia. $1: 19-57$

Sanders C.(1993). Understanding dogs: caretakers' attributions of mindedness in canine-human relationships. Journal of Contemporary

Ethnography 22(2): 205-226.

Sanders C. (2003). Actions speak louder than words: close relationships between humans and nonhuman animals. Symbolic Interaction 26: 405-426.

Travers M.(2001). Qualitative research through case studies. London: Sage.

Taylor N. (2004) 'In it for the Animals: moral certainty and animal welfare. Society \& Animals 12(4): 317-339. 
Taylor N. (2007). 'Never an It': Intersubjectivity and the creation of animal personhood, in animal shelters. Qualitative Sociology Review,3, 1: 59-73.

Wilson C. C. (1998). A conceptual framework for human-animal interaction research" (pp. 61-90), In: Wilson C.C. \& D. C. Turner Companion Animals in Human Health, Thousand Oaks: Sage. 